Cite this: Photochem. Photobiol. Sci., 2013, 12, 848

Received 22nd November 2012, Accepted 27th January 2013

DOI: $10.1039 / c 3 p p 25397 j$

www.rsc.org/pps

\section{Transient absorption spectroscopy on spiropyran monolayers using nanosecond pump-probe Brewster angle reflectometry}

\author{
Bernhard Siebenhofer, ${ }^{a, b, c}$ Sergey Gorelik, ${ }^{d}$ Martin J. Lear, ${ }^{e}$ Hong Yan Song, ${ }^{d}$ \\ Christoph Nowak ${ }^{\star a, b, f}$ and Jonathan Hobley ${ }^{* d}$
}

\begin{abstract}
Self-assembled monolayers of 11-(3',3'-dimethyl-6,8-dinitrospiro[chromene-2,2'-indoline]-1'-yl) undecanoic acid (amphiphilic spiropyran) at the air-water interface are studied using Brewster angle reflectometry. Transient kinetics of the spiropyran to merocyanine conversion are recorded in a UV-pump, VIS-probe configuration. By varying the probe wavelength using an optical parametric oscillator, we are able to reconstruct absorption spectra of intermediate states with a time-resolution of 10 nanoseconds, limited by the temporal convolution of the two laser pulses. After UV irradiation, spiropyran converts to merocyanine in two stages. The first occurs within a timescale of several tens of nanoseconds and is heavily convoluted with the system response time, whereas the second stage occurs over a few hundred nanoseconds. During the rise time there is a small red shift in the transient absorption spectrum of $\sim 20 \mathrm{~nm}$. We assign the red shift and the slower kinetics to the isomerization of a merocyanine isomer cis about the central methine bond to those that are trans about the same bond.
\end{abstract}

\section{Introduction}

Spiropyran (SP) to merocyanine (MC) conversion (Scheme 1) $)^{1-3}$ in thin films and monolayers has attracted a lot of attention in recent years because of potential applications in photoswitching devices. Studies include light-induced phase transitions that induce conformational and pressure changes, ${ }^{4-6}$ and changing the wettability of surfaces. ${ }^{7,8}$ Other works use SP photoswitching to alter surface properties for electrodes on gold to bind proteins, ${ }^{9}$ in biopolymer films ${ }^{10}$ and hybrid surface architectures. ${ }^{11}$

Despite this wide range of research, studies on the fundamental photochemical properties are scarce. This is mainly because thin films and monolayers are hard to monitor in transmittance because they are so thin that they cause only

${ }^{a}$ Austrian Institute of Technology GmbH, AIT, BioSensor Technologies, Muthgasse 11, 1190 Vienna, Austria.E-mail: hobleyj@imre.a-star.edu.sg, C.Nowak@ait.ac.at ${ }^{b}$ Centre for Biomimetic Sensor Science, CBSS, 50 Nanyang Drive, Research Technoplaza, XFrontiers Block, Singapore 637553

${ }^{c}$ University of Natural Resources and Life Sciences, BOKU, Department of Nanobiotechnology, Muthgasse 11, 1190 Vienna, Austria

${ }^{d}$ Institute of Materials Research and Engineering, ${ }^{*}$ STAR (Agency for Science, Technology and Research), 3 Research Link, Singapore 117602

${ }^{e}$ Department of Chemistry, Graduate School of Science, Tohoku University, Aoba-ku, Sendai, 980-8578, Japan

${ }^{f}$ Centre of Electrochemical Surface Technology, CEST, Viktor-Kaplan-Str. 2, 2700 Wiener Neustadt, Austria very small phase shifts in light passing through them and have inherently low absorbance due to their short pathlength. Several techniques that overcome these difficulties have been successfully applied. On metallic surfaces, surface plasmon resonance $(\mathrm{SPR})^{12-14}$ and polarization modulation Fourier transform infrared spectroscopy (PM-FTIR) can be applied. ${ }^{15}$ Waveguides $^{16}$ and cavity ring-down spectroscopy (CRDS) setups increase the optical pathlength of the sample by allowing the light to travel through the monolayers multiple

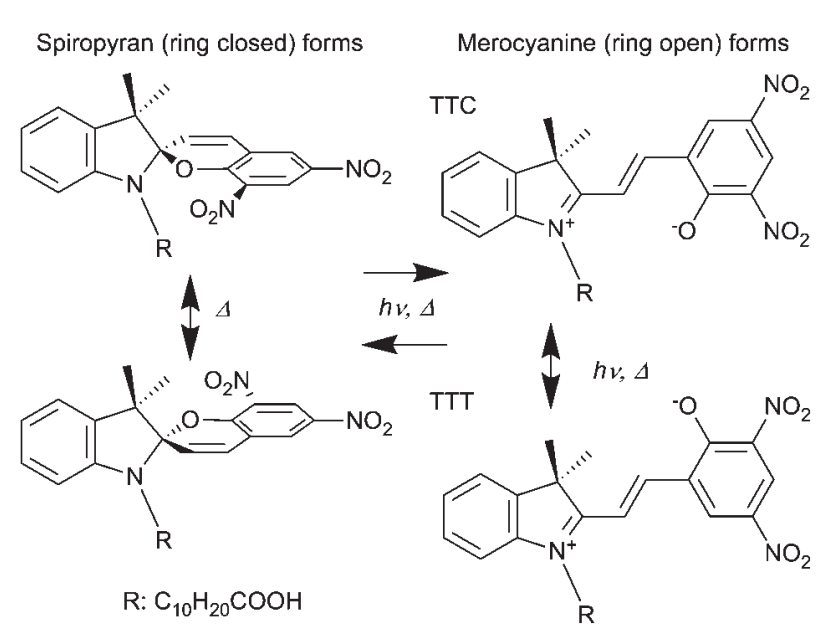

Scheme 1 
times. ${ }^{17,18}$ Ellipsometry has been used to study thin SP films. ${ }^{19,20}$

Brewster angle microscopy (BAM), a special case of ellipsometry, was independently developed by Meunier et al. and Möbius et al. as a tool to study monolayers. ${ }^{21-23}$ Therefore, it is ideally suited for monitoring SP dynamics in monolayers. BAM is based on the fact that p-polarised light is fully refracted if it is incident on a surface at Brewster's angle. If a monolayer is present at the interface of the surface then a small amount of light is reflected with an intensity dictated by the refractive index of the monolayer. Changes in the complex refractive index alter Brewster's criteria, leading to changes in the amount of light reflected. Capturing this reflected beam on a CCD camera gives high contrast images of the probed interface on an inherently dark background. Previously we have shown that BAM is a useful tool for studying photodynamics in SP thin films and monolayers. ${ }^{24,25}$

Brewster angle reflectometry (BAR), that is reflectometry in the vicinity of Brewster's angle, was used to study transient kinetics of SP monolayers on functionalised fused silica ${ }^{26}$ and self-assembled monolayers at the air-water interface. ${ }^{27}$ One drawback of the methods applied was the lack of spectral resolution in the kinetic studies due to the limitation of available probe wavelengths from the probe lasers used. Our current work expands on these techniques by measuring transient absorption kinetics at different probe wavelengths, generated by an optical parametric oscillator (OPO) pumped by a neodymium yttrium aluminium garnet (Nd-YAG) laser, in order to reconstruct spectra of transient states with nanosecond time resolution.

\section{Experimental}

\section{Brewster angle reflectometer}

Two types of BAR systems are used in this work. The primary BAR apparatus used for transient kinetic measurements has been described previously. ${ }^{27}$ The second BAR is a modified NanoFilm $\mathrm{EP}^{3}$ BAM that was used to verify monolayer conditions and measure Brewster's angle as a starting point for simulations. ${ }^{26,28}$ Both systems and recent modifications are described below.

The most notable change to the primary setup is that an OPO (Spectra-Physics MOPO-SL pumped by a Quanta-Ray Pro 270-10 pulsed Nd-YAG laser) is used as a probe light source. This allows tuning the probe light wavelength from $440 \mathrm{~nm}$ to $705 \mathrm{~nm}$ and $715 \mathrm{~nm}$ to $1850 \mathrm{~nm}$. The pulse width of this light is 4-7 ns depending on the wavelength. The probe beam is attenuated by various neutral density filters to keep it constant over the OPO tuning range. Fig. 1 shows the setup of this BAR apparatus. Briefly, a cylindrical lens focuses the probe beam (p-polarized by a high extinction, $1 \times 10^{-8}$, Glan-Taylor polarizer) into a strip on the bulk liquid surface. That way the beam has varying angles of incidence making the reflected beam diverge over this range of angles. As the approximate centre of the laser beam is incident at Brewster's angle, reflectivity will

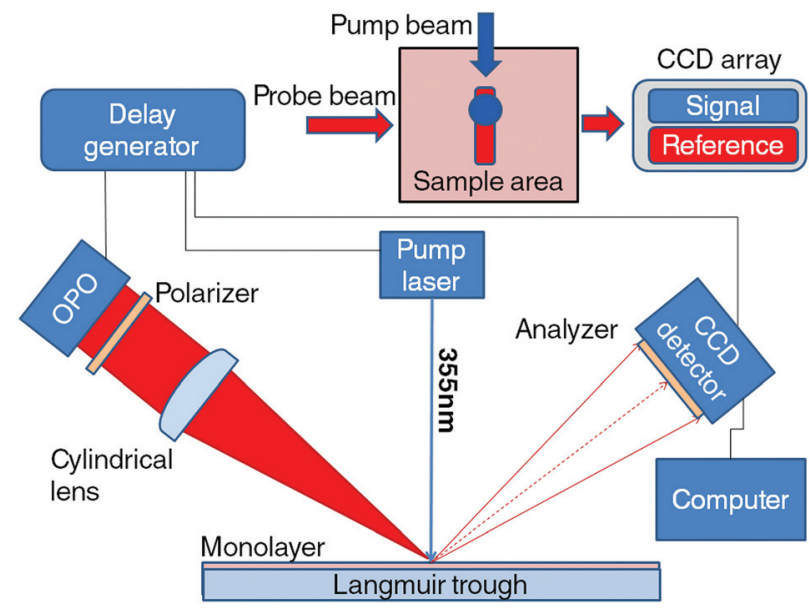

Fig. 1 Setup of the pump-probe Brewster angle reflectometer. The top right insert shows the applied method of self-referencing.

be minimized there. According to Fresnel's equations for p-polarized light, this results in a parabolic reflectivity increase around Brewster's angle. ${ }^{26}$

The reflected light is collected with a CCD camera (DU420$\mathrm{BV}$, Andor Technology, cooled to $-85^{\circ} \mathrm{C}$ for dark current suppression). The imaging area is a two dimensional array of pixels. This allows the separation of the readout to two vertical strips for self-referenced measurements. One half measures the intensity profile of probe light that is reflected from a UVpumped (354.7 nm, $8 \mathrm{~ns}$ pulse, 2nd harmonic from a Litron NANO-S-60-30 Nd-YAG laser) region of the sample. The other half monitors light from a non-irradiated part. In this way we can directly measure the shifting of Brewster's angle in a one shot self-referenced experiment. This prevents shot to shot noise due to beam profile changes or noise from vibrations in the laboratory from influencing the measurements.

The pump laser is equipped with a variable attenuator that can be used to vary the output energy between 0-10 mJ. A plano-convex lens is used to focus the pump laser to a spot size of $\sim 2 \mathrm{~mm}$ diameter on the monolayer.

Transient kinetics are measured by varying the pumpprobe delay time using two delay generators (Stanford Research Systems DG535 and Berkley Nucleonics Corporation BNC-465). The nanosecond time-resolution is limited by the temporal convolution of pump and probe laser pulses.

A photodiode (Thorlabs DET 200, $50 \Omega$ terminated, read out by a Tektronix TDS 2014B oscilloscope) is used to measure the relative output power of the pump and probe beams. In that way, we can account for shot to shot fluctuations in pump energy to improve the signal to noise ratio. Additionally it is used to verify the timing of pump and probe signals so as to correct delay time jitter.

The second system is essentially a NanoFilm $\mathrm{EP}^{3}$ BAM that is equipped with nanosecond laser excitation $(354.77 \mathrm{~nm}, 5 \mathrm{~ns}$ pulse, 2nd harmonic from a Lotis LS-2134UTF Nd-YAG laser). It is possible to make kinetic measurements with this system, however in the current work it is simply used to image the 
state (homogeneity) of the monolayer under identical conditions used for kinetic studies with the primary setup. The built-in $\mathrm{EP}^{3}$ goniometer, which has a $0.01^{\circ}$ angle resolution and a reproducibility of $0.001^{\circ}$, makes it possible to establish the Brewster angle of the monolayer very accurately. The precision of this value is important to get better estimations of monolayer thickness and initial refractive index from simulations. ${ }^{26,27}$

\section{Sample preparation}

A linear, Teflon Langmuir trough and a Hamilton microsyringe are cleaned using chloroform, ethanol, methanol, and deionised water. The spreading solution is prepared using ACS Spectro-photometric grade chloroform (stabilized with 0.5-1.0\% ethanol) supplied by Sigma Aldrich without further purification. As the bulk liquid sub-phase we use deionised water (ELAGA Lab Water, Purelab option-Q). The chloroformspiropyran spreading solution is deposited drop by drop with the needle parallel and as close as possible to the surface without actually touching it.

Amphiphilic spiropyran, 11-(3',3'-dimethyl-6,8-dinitrospiro [chromene-2,2'-indoline]-1'-yl) undecanoic acid, was synthesized from 2,3,4-trimethylindolenium 11-bromoundecanoic acid and 3,5-dinitrosalicylaldehyde via a two-step substitution and a condensation reaction as previously described. ${ }^{26,28}$

\section{Results and discussion}

\section{Theoretical considerations}

Data analysis and simulations are done analogously to previous work. ${ }^{27}$ Briefly, the reflectivity and absorption changes of the monolayer can be derived from Fresnel's equations. ${ }^{24,26,28}$ The relation between angular shifts and absorption is linear for initially transparent monolayers. Our simulations and calculations have shown that it is possible to independently determine the changes of the real and imaginary part of the complex refractive index

$$
\tilde{n}=n+\mathrm{i} \kappa
$$

of the monolayer from the behaviour of Brewster's angle minima. Angular shifts are linear in respect to changes of $\kappa$, while changes in measured intensity at Brewster's angle are mainly caused by changes in $n$.

Absorbances are then calculated from $\kappa$ values using BeerLambert's law ${ }^{29}$

$$
A b s=\frac{4 \pi}{\lambda} \kappa d
$$

where $d$ is the thickness of the monolayer and $\lambda$ is the probe wavelength. As absorbances are usually supplied in decadic logarithmic base this value will be divided by 2.303 .

\section{Monolayer kinetics and time resolved spectra}

Fig. 2 shows the kinetics of absorbance changes of a monolayer with a surface area of $0.29 \mathrm{~nm}^{2}$ per spiropyran molecule.

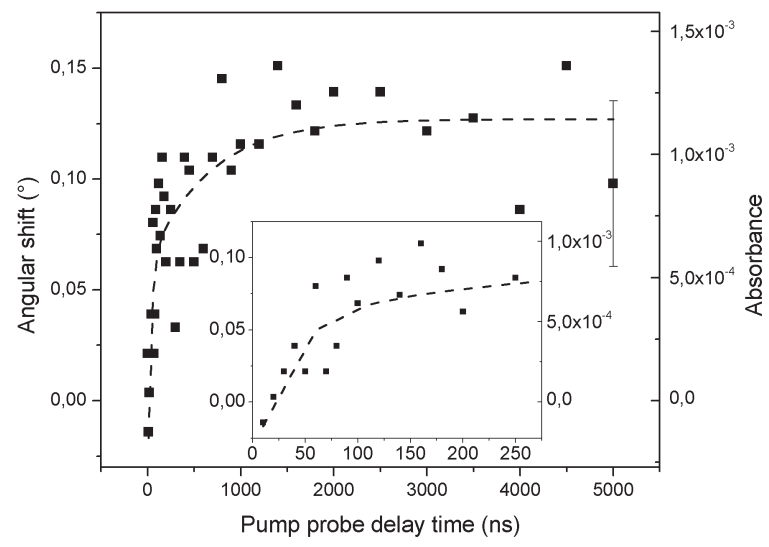

Fig. 2 Kinetic trace of the absorbance change in a spiropyran monolayer probed at $555 \mathrm{~nm}$ with its biexponential fit. The error bar of the last data point indicates the maximal expected statistical error, estimated from pre $t_{0}$ measurements under same conditions. Axis legends of the insert are identical to the main graph.

For this measurement the OPO was set to $555 \mathrm{~nm}$, the maximum of the merocyanine absorbance peak in chloroform solution (Fig. 3). From biexponential fitting

$$
A=A_{0}-A_{1} e^{\frac{\tau}{\tau_{1}}}-A_{2} e^{-\frac{\tau}{\tau_{2}}}
$$

we find a fast, $\tau_{1}=33 \pm 11 \mathrm{~ns}$, and a slow, $\tau_{2}=650 \pm 217 \mathrm{~ns}$, component in the conversion kinetics. The amplitude of the fast component $A_{1}$ is twice as big as that of the slow component $A_{2}$. The fact that the total absorbance is lower than the previous results for kinetics at $532 \mathrm{~nm}$ suggests that the merocyanine peak is blue shifted in a monolayer on water compared to chloroform solution. This solvatochromism can be attributed to the higher polarity of water. ${ }^{1,30}$ This blue shift is confirmed in the current work (vide infra).

Combining kinetic traces collected with different probe wavelengths allows us to reconstruct transient spectra at different pump-probe delay times. Fig. 4 shows the monolayer spectrum at $1 \mu$ s pump-probe delay time obtained with this

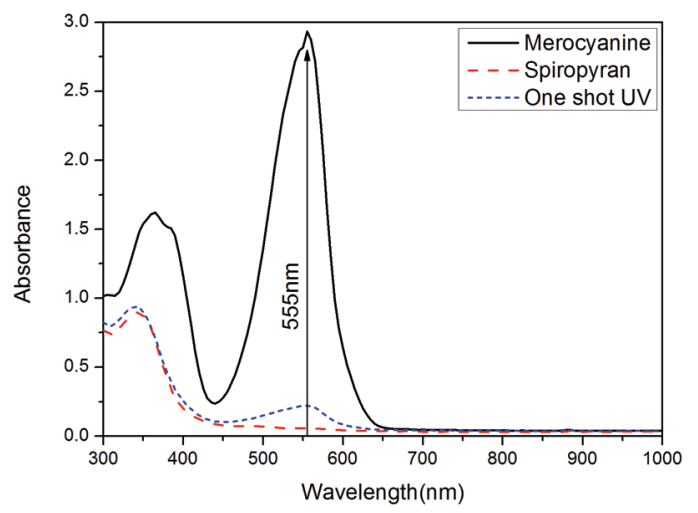

Fig. 3 UV-Vis spectra of dark adapted merocyanine, completely photobleached spiropyran and the merocyanine state after irradiation with one $10 \mathrm{~mJ}$ pump pulse at $355 \mathrm{~nm}$. Measurements are made in a $0.5 \mathrm{~cm}$ pathlength quartz cuvette. 


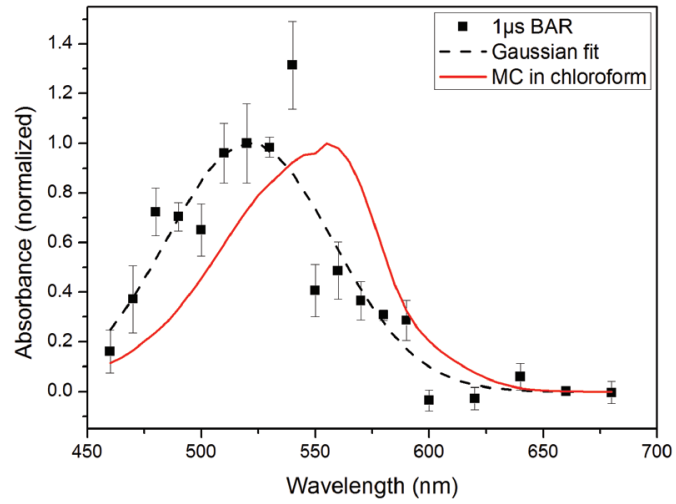

Fig. 4 Comparison of normalized UV-Vis and BAR spectra. The BAR spectrum is measured at a delay time of $1 \mu \mathrm{s}$. Each data point shown is the average of five measurements with its standard error.

method compared to a spectrum obtained in chloroform. The absorption maximum is estimated by fitting a single Gaussian peak to the spectrally resolved data. Comparing these two spectra we find a solvatochromatic blue shift of about $35 \mathrm{~nm}$, which is consistent with the merocyanine being in a more polar environment. ${ }^{1}$

Fig. 5 shows the transient absorption spectra at different pump-probe delay times. Following the growth of the merocyanine peak from the system response time ( $20 \mathrm{~ns}$ ) to the plateau region $(1 \mu \mathrm{s})$, we observe a gradual red shift with prolonged pump-probe delay times. This red shift amounts to $\sim 20 \mathrm{~nm}$ from the $100 \mathrm{~ns}$ pump-probe delay time to the saturated conversion. It is not possible to fit peaks to the data from pumpprobe delay times below $100 \mathrm{~ns}$. This is because the expected peak is supposedly smaller than the statistical error of our measurements.

From these results, we can conclude that the conversion of spiropyran to the final merocyanine composition occurs in two stages. Previously, it was proposed that the slower component may be the result of the isomerization of the ring-open merocyanine cis about the central methine bond to merocyanine

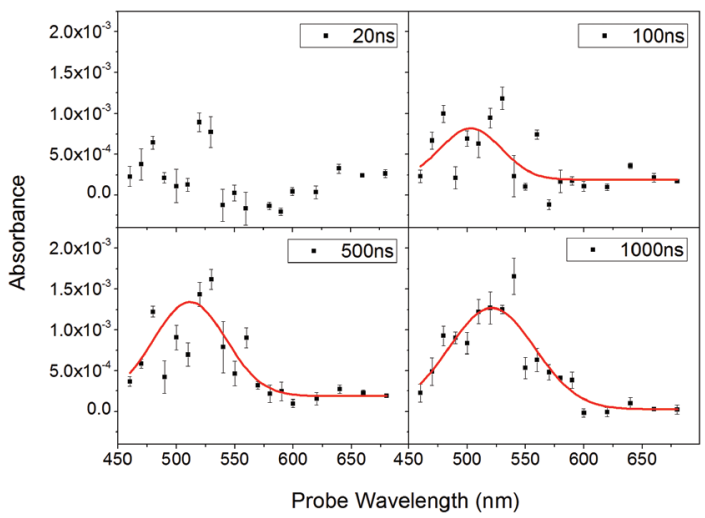

Fig. 5 Transient absorption spectra at different pump-probe delay times between the lower limit of $20 \mathrm{~ns}$ and the plateau region after $1 \mu \mathrm{s}$. Each data point is the mean of five measurements with its standard error. Lines are Gaussian fits that indicate the expected peak area. isomers that are trans about this bond. ${ }^{27}$ However, from NMR data we also know that there are two isomers (TTC and TTT) for this particular molecule in chloroform solution, with more than $70 \%$ TTC in equilibrium. ${ }^{1,2}$ This ratio of TTC to TTT is in good agreement with the two amplitudes of the double exponential fit discussed in Fig. 2. Therefore the second component of the kinetics could, in principle, be due to cis-trans conversions between TTC and TTT. However, the interconversion between these two forms occurs on a timescale of $10 \mathrm{~ms}$ at $243 \mathrm{~K},{ }^{2}$ so it should be too slow to account for the current observations.

The latter rise is associated with a spectral shift of $20 \mathrm{~nm}$, which is a little less than is observed for similar cis-trans conversions in stilbene $(\sim 30 \mathrm{~nm})^{31}$ and the $\pi-\pi^{*}$ transition of azobenzene $(\sim 40 \mathrm{~nm}) .{ }^{32}$ For chromene derived mero-forms it has been observed that a mixture of isomers trans about the terminal methine bond, but trans and cis about the terminal methine bond, has a red shift of $\sim 7 \mathrm{~nm}$ compared to the pure trans component. ${ }^{33}$ Similar shifts have been observed for cistrans isomerizations in terminal methine bonds of spiropyran derived merocyanines. ${ }^{34}$

In the current case, in view of the observed spectral shifts and with knowledge of the isomerization rate between the TTC and TTT isomers of $\sim 10 \mathrm{~ms},{ }^{2}$ we consider that the $\sim 20 \mathrm{~nm}$ shift currently observed is most likely due to isomerization from an isomer cis about the central methine to one trans about that bond. In any event, we can state with some confidence, based upon our spectroscopically resolved data, that the longer component of the kinetic traces seen presently and previously ${ }^{27}$ is associated with the red shift. Furthermore, this red-shift is consistent with merocyanine isomerization reactions that are slower in the monolayer, taking $100 \mathrm{~s}$ of nanoseconds compared to a solution where similar transformations take a few picoseconds. ${ }^{35}$ We propose that this retardation is due to constricted motion and geometry in the densely packed monolayer.

Fig. 6 shows absorbance and angular shifts for different pump laser fluences. Measurements are taken using $555 \mathrm{~nm}$ probe light. By normalising the angular shifts to a pump

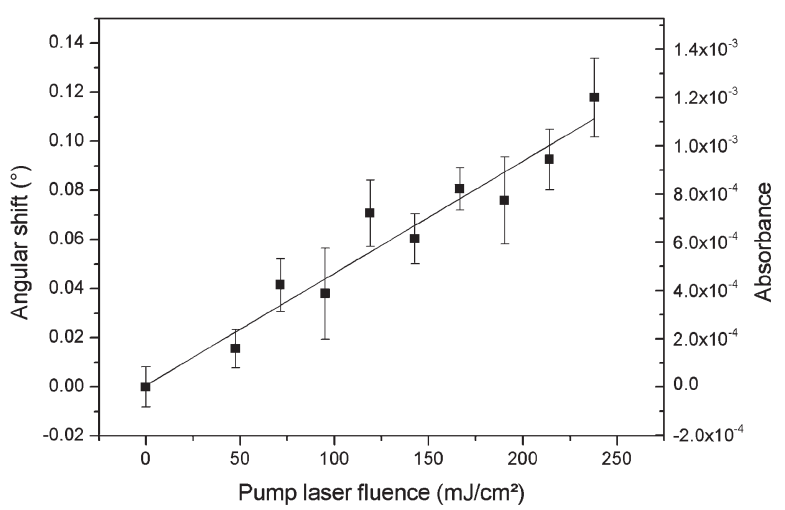

Fig. 6 Angular shift and corresponding absorbance at $1 \mu$ s pump-probe delay time for $555 \mathrm{~nm}$ probe light for different pump laser fluences. Statistics are mean and standard error of five measurements at each data point. 
power output, we could significantly decrease deviations from the linear fit compared to previous results. ${ }^{27}$ It is worth commenting on the rather high fluences that the monolayer seems to withstand without apparent damage or saturation of the photochemical conversion process. From the measured transient absorbance, surface coverage and theoretical merocyanine absorbance, ${ }^{1}$ we estimate a conversion rate of $\sim 9 \%$ at $250 \mathrm{~mJ}$ $\mathrm{cm}^{-2}$. This is consistent with conversions achievable in chloroform solution. Generally, we can state that the quantum yield for coloration of this particular type of spiropyran is very low ${ }^{28,36,37}$ and this explains the high fluences required to achieve this conversion. This is opposite, yet analogous, to the case of spirooxazine ring closure where Bohne et al. used laser fluences up to $600 \mathrm{~mJ}$ per pulse to photobleach merocyanine isomers due to the very low quantum yield of this process. ${ }^{38}$

\section{Conclusions}

By using the variable wavelength output of an OPO system, we have taken the step from measuring transient angle-resolved reflectivity changes at single wavelengths to collecting absorption spectra over the whole visible region. This method allows the spectroscopic assessment of transient states in monolayers at the air-water interface. Nanosecond time resolution is only limited by the temporal convolution of the OPO and pump laser pulses.

Monolayers of amphiphilic spiropyran convert to merocyanine after UV irradiation. Qualitative measurements have shown that the merocyanine peak red-shifts gradually by $\sim 20 \mathrm{~nm}$ during its rise time. We attribute this shift to isomerization of an intermediate that is cis about the central methine bond to the final merocyanine isomers that are trans about this bond. The isomerization is slower than that observed in solution due to constricted geometry in the densely packed monolayer.

\section{Acknowledgements}

This work was funded by the International Graduate School Bio-Nano-Tech, a joint PhD program of University of Natural Resources and Life Sciences Vienna (BOKU), the Austrian Institute of Technology (AIT) (GZ BMVIT-612.166/0001-III/I1/2010), A-STAR Graduate Academy and the Nanyang Technological University (NTU). Equipment and materials were supported by A-STAR JCO and A-STAR-JST.

\section{Notes and references}

1 J. Hobley, V. Malatesta, R. Millini, L. Montanari and W. O. N. Parker, Proton exchange and isomerisation reactions of photochromic and reverse photochromic spiropyrans and their merocyanine forms, Phys. Chem. Chem. Phys., 1999, 1, 3259-3267.
2 J. Hobley and V. Malatesta, Energy barrier to ttc-ttt isomerisation for the merocyanine of a photochromic spiropyran, Phys. Chem. Chem. Phys., 2000, 2, 57-59.

3 J. Hobley, U. Pfeifer-Fukumura, M. Bletz, T. Asahi, H. Masuhara and H. Fukumura, Ultrafast photo-dynamics of a reversible photochromic spiropyran, J. Phys. Chem. A, 2002, 106, 2265-2270.

4 K. Balashev, I. Panaiotov and J. E. Proust, Propagation of photoinduced surface pressure perturbation along a mixed benzospiropyran-octadecanol monolayer, Langmuir, 1997, 13, 5373-5377.

5 H. Tachibana, Y. Yamanaka, H. Sakai, M. Abe and M. Matsumoto, J-aggregate formation of amphiphilic merocyanine in Langmuir-Blodgett films, J. Lumin., 2000, 87-89, 800-802.

6 M. Matsumoto, Photoreactions and lateral patterning in Langmuir and Langmuir-Blodgett films, Chem. Rec., 2007, 7, 69-77.

7 R. Rosario, D. Gust, M. Hayes, F. Jahnke, J. Springer and A. A. Garcia, Photon-modulated wettability changes on spiropyran-coated surfaces, Langmuir, 2002, 18, 8062-8069.

8 S. Wang, Y. Song and L. Jiang, Photoresponsive surfaces with controllable wettability, J. Photochem. Photobiol., C, 2007, 8, 18-29.

9 E. Katz, B. Willner and I. Willner, Light-controlled electron transfer reactions at photoisomerizable monolayer electrodes by means of electrostatic interactions: active interfaces for the amperometric transduction of recorded optical signals, Biosens. Bioelectron., 1997, 12, 703-719.

10 B. Mecheri, P. Baglioni, O. Pieroni and G. Caminati, Molecular switching in nano-structured photochromic films of biopolymers, Mater. Sci. Eng., C, 2003, 23, 893-896.

11 E. Aznar, R. Casasús, B. García-Acosta, M. D. Marcos, R. Martínez-Máñez, F. Sancenón, J. Soto and P. Amorós, Photochemical and chemical two-channel control of functional nanogated hybrid architectures, Adv. Mater., 2007, 19, 2228-2231.

12 S.-H. Kim, H.-J. Suh, K.-N. Koh, S.-A. Suck, H.-J. Choi and H.-S. Kim, Surface plasmon resonance study on the interaction of photochromic spironaphthoxazine with l-phenylalanine in self-assembled monolayers on gold, Dyes Pigm., 2004, 62, 93-97.

13 K. Sasaki and T. Nagamura, Ultrafast all-optical switch using complex refractive index changes of thin films containing photochromic dye, Appl. Phys. Lett., 1997, 71, 434-436.

14 R. B. M. Schasfoort and A. J. Tudos, Handbook of surface plasmon resonance, RCS Publishing, Cambridge, UK, 2008.

15 B. L. Frey, R. M. Corn and S. C. Weibel, Polarization-modulation approaches to reflection absorption spectroscopy, in Handbook of vibrational spectroscopy, ed. J. Chalmers and P. R. Griffiths, John Wiley \& Sons, Chichester, UK, 2001, pp. 1042-1056.

16 H. Kawai, K. Nakano and T. Nagamura, White light optical waveguide detection of transient absorption spectra in ultrathin organic films upon pulsed laser excitation, Chem. Lett., 2001, 30, 1300-1301. 
17 R. N. Muir and A. J. Alexander, Structure of monolayer dye films studied by Brewster angle cavity ringdown spectroscopy, Phys. Chem. Chem. Phys., 2003, 5, 1279-1283.

18 I. M. P. Aarts, B. Hoex, A. H. M. Smets, R. Engeln, W. M. M. Kessels and M. C. M. v. d. Sanden, Direct and highly sensitive measurement of defect-related absorption in amorphous silicon thin films by cavity ringdown spectroscopy, Appl. Phys. Lett., 2004, 84, 3079-3081.

19 Y. G. Mo, R. O. Dillon, P. G. Snyder and T. E. Tiwald, Optical properties of photochromic organic-inorganic composites, Thin Solid Films, 1999, 355, 1-5.

20 Y. G. Mo, R. O. Dillon and P. G. Snyder, Spectroscopic analysis of photochromic films, J. Vac. Sci. Technol., A, 1999, 17, 170-175.

21 S. Henon and J. Meunier, Microscope at the Brewster angle: direct observation of first-order phase transitions in monolayers, Rev. Sci. Instrum., 1991, 62, 936-939.

22 D. Hönig and D. Möbius, Direct visualization of monolayers at the air-water interface by Brewster angle microscopy, J. Phys. Chem., 1991, 95, 4590-4592.

23 D. Hönig and D. Möbius, Reflectometry at the Brewster angle and Brewster angle microscopy at the air-water interface, Thin Solid Films, 1992, 210-211(Part 1), 64-68.

24 J. Hobley, T. Oori, S. Gorelik, S. Kajimoto, H. Fukumura and D. Honig, Time-resolved Brewster angle microscopy for photochemical and photothermal studies on thinfilms and monolayers, J. Nanosci. Nanotechnol., 2009, 9, 59-68.

25 J. Hobley, T. Oori, S. Kajimoto, S. Gorelik, D. Honig, K. Hatanaka and H. Fukumura, Laser-induced phase change in Langmuir films observed using nanosecond pump-probe Brewster angle microscopy, Appl. Phys. A: Mater. Sci. Process., 2008, 93, 947-954.

26 S. Gorelik, S. Hongyan, M. J. Lear and J. Hobley, Transient Brewster angle reflectometry of spiropyran monolayers, Photochem. Photobiol. Sci., 2010, 9, 141-151.

27 B. Siebenhofer, S. Gorelik, A. V. Sadovoy, M. J. Lear, H. Y. Song, C. Nowak and J. Hobley, Photoconversion of spiropyran to merocyanine in a monolayer observed using nanosecond pump-probe Brewster angle reflectometry, Aust. J. Chem., 2012, 65, 283-289.

28 S. Gorelik, J. Hobley, M. J. Lear, H. Song, S. Y. S. Yong and J. Cheng, Quantification of transient absorption in photoreactive monolayers using reflectometry in the vicinity of Brewster angle, Int. J. Nanosci., 2009, 08, 213.

29 H. Fujiwara, Spectroscopic ellipsometry: principles and applications, John Wiley \& Sons, Ltd, West Sussex, England, 2007.

30 W. Liptay, Electrochromism and solvatochromism, Angew. Chem., Int. Ed. Engl., 1969, 8, 177-188.

31 S. Yamashita, Photochemical cis-trans isomerization of stilbene, Bull. Chem. Soc. Jpn., 1961, 34, 4.

32 G. Gabor and E. Fischer, Spectra and cis-trans isomerism in highly dipolar derivatives of azobenzene, J. Phys. Chem., 1971, 75, 581-583.

33 J. Hobley, V. Malatesta, K. Hatanaka, S. Kajimoto, S. L. Williams and H. Fukumura, Picosecond and nanosecond photo-dynamics of a naphthopyran merocyanine, Phys. Chem. Chem. Phys., 2002, 4, 180-184.

34 T. Horii, Y. Miyake, R. Nakao and Y. Abe, Another photochromism of a spironaphtoxazine due to the geometric isomerization of the open-form, Chem. Lett., 1997, 26, 655-656.

35 N. Tamai and H. Miyasaka, Ultrafast dynamics of photochromic systems, Chem. Rev., 2000, 100, 1875-1890.

36 M. Bletz, U. Pfeifer-Fukumura, U. Kolb and W. Baumann, Ground- and first-excited-singlet-state electric dipole moments of some photochromic spirobenzopyrans in their spiropyran and merocyanine form, J. Phys. Chem. A, 2002, 106, 2232-2236.

37 D. A. Reeves and F. Wilkinson, Photochromism of spiropyrans. Part 1.-mechanism of photocolouration, J. Chem. Soc., Faraday Trans. 2, 1973, 69, 1381-1390.

38 C. Bohne, M. G. Fan, Z. J. Li, J. Lusztyk and J. C. Scaiano, Photochromic processes in spiro(1,3,3-trimethylindolo-2,2[primeor minute]-naphth[1,2-b]-1,4-oxazine) studied using two-laser two-colour techniques, J. Chem. Soc., Chem. Commun., 1990, 571-572. 